\title{
Biomarkers for immunotherapy for treatment of glioblastoma
}

John P Lynes, Anthony K Nwankwo, Hannah P Sur, Victoria E Sanchez, Kwadwo A Sarpong, Oluwatobi I Ariyo, Gifty A Dominah, Edjah K Nduom
To cite: Lynes JP, Nwankwo AK, Sur HP, et al. Biomarkers for immunotherapy for treatment of glioblastoma. Journal for ImmunoTherapy of Cancer 2020;8:e000348. doi:10.1136/ jitc-2019-000348

JPL and AKN contributed equally.

JPL and AKN are joint first authors.

Accepted 09 April 2020
Check for updates

(c) Author(s) (or their employer(s)) 2020. Re-use permitted under CC BY-NC. No commercial re-use. See rights and permissions. Published by BMJ.

National Institute of Neurological Disorders and Stroke, National Institutes of Health, Bethesda, Maryland, USA

Correspondence to

Dr Edjah K Nduom;

edjah.nduom@nih.gov

\section{ABSTRACT}

Immunotherapy is a promising new therapeutic field that has demonstrated significant benefits in many solid-tumor malignancies, such as metastatic melanoma and nonsmall cell lung cancer. However, only a subset of these patients responds to treatment. Glioblastoma (GBM) is the most common malignant primary brain tumor with a poor prognosis of 14.6 months and few treatment advancements over the last 10 years. There are many clinical trials testing immune therapies in GBM, but patient responses in these studies have been highly variable and a definitive benefit has yet to be identified. Biomarkers are used to quantify normal physiology and physiological response to therapies. When extensively characterized and vigorously validated, they have the potential to delineate responders from nonresponders for patients treated with immunotherapy in malignancies outside of the central nervous system (CNS) as well as GBM. Due to the challenges of current modalities of radiographic diagnosis and disease monitoring, identification of new predictive and prognostic biomarkers to gauge response to immune therapy for patients with GBM will be critical in the precise treatment of this highly heterogenous disease. This review will explore the current and future strategies for the identification of potential biomarkers in the field of immunotherapy for GBM, as well as highlight major challenges of adapting immune therapy for CNS malignancies.

\section{INTRODUCTION}

Immune-based therapies harness a patient's immune system to specifically target and destroy cancer cells. ${ }^{1}$ The field of immunotherapy has already made significant developments in the treatment of several aggressive cancer types, including melanoma, ${ }^{2}$ non-small cell lung cancer (NSCLC) ${ }^{3}$ and lymphomas. ${ }^{45}$ However, despite advances in immune therapy, there is an accompanying need for the development of biomarkers that accurately measure a patient's response to therapy.

Biomarkers are quantifiable factors that gauge normal physiology, pathology, treatment response or biological responses to intervention. ${ }^{6}$ Relevant biomarkers can be used to determine which patients have effectively responded to immune therapy, identify risks and recurrences or even predict which patients are most likely to benefit from a particular treatment regimen. ${ }^{7}$

While immunotherapy offers some hope in the management of other cancers, it has not yet demonstrated improved overall survival (OS) for patients with glioblastoma (GBM) ${ }^{8-10} \mathrm{GBM}$, the most common malignant primary brain tumor in adults, ${ }^{11} 12$ is known for its poor median survival, high recurrence rate and limited treatment advancements. ${ }^{13}$ The intratumoral molecular heterogeneity and immunosuppressive tumor microenvironment of GBM are partly responsible for its therapeutic resistance and recurrence. ${ }^{14-18}$ Key dysregulations of the immune system within the tumor microenvironment, and this cancer's anatomical location within the central nervous system (CNS) make the discovery of biomarkers to measure response to immune therapy particularly important.

Even in immune therapy trials reporting positive results, such as for untreated melanoma, ${ }^{2}$ only a subset of patients receives benefit from treatment. Similar mixed results have been reported in most immune therapy trials for various malignancies. High variability in patients' clinical responses highlights the need for biomarkers which could guide clinicians to more precise treatment options based on each patient's molecular and immune-related profiles. ${ }^{19}$

This review will examine current strategies and future directions in the discovery of GBMspecific biomarkers for immune therapy, major challenges that limit the ability to effectively adapt immunotherapy treatment in the CNS and highlight the potential role that genetic sequencing and other innovative technologies will have in discovering GBMspecific biomarkers.

\section{BIOMARKERS IN NON-CNS CANCERS AND THE DETERMINATION OF RESPONSES TO IMMUNE THERAPY}

Several biomarkers, such as the presence of a pro-inflammatory $\mathrm{T}$ cell phenotype and 
molecular alterations in the tumor microenvironment, have made progress in helping to determine how patients will respond to checkpoint inhibition therapy in other cancers. ${ }^{2021}$ However, most solid tumors exhibit molecular and immunological heterogeneity, ${ }^{22}$ which creates challenges in identifying biomarkers that determine which patients will benefit from immunotherapy. Tissue sampling, particularly of tumor tissue, remains the gold standard of pathological diagnosis and is critical for the evaluation and corroboration of most biomarkers under investigation. Tissue sampling is particularly important after treatment, as radiographic evidence of progression may not be reflected on tissue analysis. ${ }^{23}$

\section{Immunophenotyping in non-CNS malignancy}

Immunophenotyping, the characterization of the immune system components within tissue, has been a common technique for identifying potential biomarkers. This methodology focuses on cell type identification and analysis via antibody targeting such as immunohistochemistry (IHC). It is widely used in preclinical and clinical studies to investigate the immune system's relationship with malignancy.

Lymphocytes, particularly $\mathrm{T}$ cells, were identified in tumors, blood and lymph nodes of patients with melanoma decades ago. ${ }^{24}$ Since this discovery, tumor infiltrating lymphocytes (TILs) and tumor-associated macrophages (TAMs) have been associated with disease prognosis in many solid tumors. ${ }^{25-28}$ In particular, $\mathrm{CD} 103^{+}$ lymphocytes, most of which are $\mathrm{CD} 8^{+} \mathrm{T}$ cells, have been reported as a predictive biomarker of improved response with checkpoint inhibition therapy. ${ }^{29-31}$ Conversely, the presence of immunosuppressive cell types, such as regulatory $\mathrm{T}$ cells (Tregs), has been described as a negative prognostic biomarker in a variety of malignancies. ${ }^{32-34}$ Other groups report that the ratio of immunosuppressive and inflammatory cell types are prognostic biomarkers, with an increased ratio of Tregs cells to $\mathrm{CD} 8^{+}$cytotoxic T lymphocytes (CTLs) portending a poor prognosis. ${ }^{35} 36$

Studies with available pre-treatment and post-treatment tissue samples have helped to describe the effects of immune therapeutics on the tumor immune microenvironment of many cancers. These tissue-based pathological responses can then be related to survival and progression data from clinical trials to search for clinical correlation.

As study results of immunotherapies are released and recurrences are analyzed, there has been more data regarding pathological response following immune therapy. A phase II trial investigating imiquimod, an immunotherapy that activates toll-like receptor 7 on macrophages to increase the secretion of pro-inflammatory cytokines, in vulvar intraepithelial neoplasia demonstrated that the frequency of intratumoral $\mathrm{CD} 4^{+}$and $\mathrm{CD} 8^{+}$ cells increased in response to treatment in responders, while non-responders demonstrated elevated Tregs. ${ }^{37} 38$ Notably, there was no significant difference in CD4 and CD8 levels in pre-treatment biopsy that would act as a predictive marker.
Lui et alreported improved survival in a subset of B16F10 melanoma-bearing mice with anti-PD-1 (programmed death-1) immunotherapy. On stratifying their population into responders and non-responders based on survival differences, they noticed the interferon (IFN)- $\gamma$ secretion kinetics of peripheral lymphocytes could be used as an accurate predictive biomarker of response to this treatment. $^{22}$

Chen et al performed a longitudinal study of patients with melanoma with multiple biopsies dictated by treatment response after serial checkpoint inhibitors of cytotoxic $\mathrm{T}$ lymphocyte associated protein-4 (CTLA-4) followed by PD-1 blockade. ${ }^{19}$ Patients underwent biopsy prior to initiation of anti-CTLA-4 therapy. Non-responders were re-biopsied prior to initiation of anti-PD-1 therapy. On-treatment biopsies were also obtained. Pre-treatment biopsies did not show any immune cell population differences between responders and non-responders, but early on-treatment biopsies showed higher $\mathrm{CD}^{+}$cell populations in responders to anti-CTLA-4. Pre-treatment biopsies prior to PD-1 blockade showed mostly higher $\mathrm{CD}^{+}, \mathrm{CD}^{+}$and $\mathrm{CD} 45 \mathrm{RO}^{+}$cells in subsequent treatment responders. Early on-treatment biopsies of these patients' tumors showed large increases in CD3, CD4, CD8, PD-1, PD-L1, LAG-3 and FoxP3 in responders. This study provided a rare view of predictive biomarkers and potential biomarkers of treatment response in longitudinal samples.

\section{Programmed death-ligand 1 as an immune biomarker in non- CNS malignancy}

Program death ligand-1 (PD-L1) is the ligand for PD-1, an immunosuppressive immune checkpoint that has been successfully targeted by multiple checkpoint inhibitors. ${ }^{39}$ Several clinical trials have analyzed the expression of PD-L1 in the tumor microenvironment as both a marker of prognosis for the tumors as well as to predict response to anti-PD-1/PD-L1 checkpoint inhibition. ${ }^{40-43}$ In a multicenter phase II, single-arm clinical trial, 270 patients with metastatic urothelial carcinoma were administered nivolumab, an anti-PD-1 antibody, to assess its safety and efficacy. ${ }^{44}$ They discovered that objective response was achieved in $28.4 \%$ of patients with PD-L1 expression $>5 \%$, in $23.8 \%$ of patients with PD-L1 expression $>1 \%$ and in only $16.1 \%$ in patients with PD-L1 expression $<1 \%$. Conversely, in a randomized, phase III study designed to evaluate the safety and efficacy of nivolumab in 272 patients with advanced squamous cell NSCLC, ${ }^{45}$ they did not find any prognostic or predictive value of PD-L1 expression with nivolumab treatment. Due to these conflicting results, PD-L1 is not routinely used as a predictive biomarker in these patients.

\section{Genetic biomarkers in non-CNS malignancy}

Genetic profiling has demonstrated potential in discovering biomarkers in cancer. Das et al used genetic profiling to examine the immune response of tumors to checkpoint inhibition. ${ }^{46}$ When analyzing blood and 
tumor tissue from 45 patients undergoing checkpoint blockade, they found that combination therapy with antibodies targeting PD-1 and CTLA-4 led to an increase in $\mathrm{T}$ cell genes differentially expressed and a robust upregulation of IFN- $\gamma$. IFN- $\gamma$ has been validated by numerous studies to be a good predictive biomarker in many solid tumors. ${ }^{47-49}$ Furthermore, Das et al showed that anti-PD-1 therapy induced cell lysis and expression of natural killer (NK) genes on T cells. A similar finding was reported in patients with melanoma. ${ }^{50}$ Gao et al showed that loss of IFN- $\gamma$ in mice with melanoma was associated with poorer therapy response, further supporting the role of IFN $\gamma$ in antitumor response and long-term survival. ${ }^{51}$

Aside from identification of specific mutations, mutational burden determined by somatic genomic sequencing has been investigated in various malignancies to determine whether this can predict a response to checkpoint inhibition. ${ }^{52}$ High mutational burden has been associated with greater therapeutic response to immune therapy in non-CNS malignancies, particularly NSCLC and melanoma, among others. ${ }^{53} 54$ Mutational burden has been particularly supported as a predictive biomarker of clinical benefit for patients with NSCLC on immune therapy, ${ }^{556}$ despite it not being prognostic of survival for patients not on immune therapy. ${ }^{57}$

Goodman et al assessed the effects of tumor mutational burden (TMB) and clinical outcomes following immunotherapy in a retrospective review of 1638 patients with cancer, and reported a positive correlation between higher TMB and responsiveness to anti-PD-1/PD-L1 therapy in melanoma, NSCLC and many other tumors. ${ }^{58}$ This similar finding was reported in patients with colorectal cancer. ${ }^{59}$ Thus, clinical trials ${ }^{60}$ have sought to identify molecular alterations in cancers from patients who are classified as exceptional responders. ${ }^{61}$ Recent technological advancements have allowed the use of targeted sequencing of genes associated with increased mutational burden which could streamline the process of using mutational burden as a biomarker of response. ${ }^{62}$

\section{Immunoscore in non-CNS malignancies}

The Immunoscore is a newly developed pathological prognostic indicator which may develop into a predictive biomarker for immune therapy. Galon et al examined the density and location of specific infiltrative immune cells in patients with colorectal cancer and discovered that these factors were better predictors of OS than traditional histopathological methods. ${ }^{63}$ These findings have led to an additional system of cancer staging known as the Immunoscore. This system combines the traditional metrics used in the tumor, node, metatasis classification system, which primarily looks at the anatomical extent of disease for cancer staging, ${ }^{64}$ and a new component that evaluates the density of immune cells in the infiltrating tumor margin and the tumor core.$^{65}$ This system has been used extensively in hepatocellular carcinoma, melanoma, colorectal, high-grade serous ovarian, invasive breast and gastric cancer. ${ }^{66-76}$ By routinely calculating an
Immunoscore at the time of diagnosis prior to treatment with immune therapy, we may find that the Immunoscore can predict responses to checkpoint inhibition.

\section{Biomarkers of response in metastatic CNS malignancies}

Recent developments in checkpoint blockade have highlighted potential differences in response to immunotherapy between primary GBM and metastatic brain tumors. These clinical trials have demonstrated survival benefits for patients with tumors that frequently metastasize to the brain such as melanoma and NSCLC. ${ }^{77} 78$ The CheckMate-204 trial, combining nivolumab and ipilimumab, reported significant intracranial response rates of $46 \%$ in melanoma brain metastases. ${ }^{79}$ Predictive biomarkers in metastatic CNS malignancies have also been investigated by researchers. Capone et al looked at baseline neutrophil-to-lymphocyte ratio (NLR) as a predictive biomarker of response to nivolumab treatment in patients with advanced melanoma. ${ }^{80}$ Their group examined 97 patients with stage IV melanoma and discovered that absolute neutrophil count, NLR, derived neutrophilto-lymphocyte ratio (dNLR) and lactate dehydrogenase were significantly associated with survival. More importantly, however, a subgroup analysis of 27 patients with brain metastases showed that NLR $<4.7$ and dNLR $<3.8$ were associated with extended OS and progression-free survival (PFS). A study by Berghoff et al used Immunoscore to evaluate the TILs in 116 brain metastases samples from melanoma, renal cell carcinoma and lung cancer. ${ }^{81}$ This study showed a strong positive correlation between median $\mathrm{OS}$ and $\mathrm{CD}^{+}, \mathrm{CD}^{+}$and $\mathrm{CD} 45 \mathrm{RO}^{+}$TIL density.

\section{BIOMARKERS IN GLIOBLASTOMA}

A primary obstacle to the development of immunotherapy for GBM is the lack of accurate measures of treatment response. The Immunotherapy Response Assessment for Neuro-Oncology (iRANO), criteria used to determine if patients respond to immune therapy, relies on MRI to differentiate responders from nonresponders. ${ }^{82}$ However, imaging alone cannot distinguish tumor progression from immunotherapy-induced inflammatory changes. ${ }^{83} 84$ Although the use of tissue sampling of a potential recurrence to guide treatment poses risks to patients, it remains the gold standard for the diagnosis of progression versus pseudoprogression. Moreover, there are relatively few published trial results of pathological changes in checkpoint inhibition-treated GBMs. Nonetheless, the development of new biomarkers could both help identify future responders and obviate the ongoing need for tissue confirmation of progression.

\section{Immunophenotyping to determine biomarkers}

As in other malignancies, immunophenotyping has been widely used in preclinical and clinical studies of GBM. In an immune-competent murine model of GBM, Zeng et al found that the combination of anti-PD-1 therapy and radiation increased the $\mathrm{CD}^{+}$Tcell-to-Treg ratio 
in responders. ${ }^{85}$ Similarly, Fecci et al demonstrated that CTLA-4 blockade enhanced $\mathrm{CD} 4^{+} \mathrm{T}$ cell proliferative capacity and mitigated Treg-meditated suppression of these cells in murine models. ${ }^{86}$ Conversely, temozolomide increases the proportion of exhausted $\mathrm{T}$ cells in mice with intracranially implanted gliomas, and this reduces their response to checkpoint inhibition. ${ }^{87}$ This finding suggests that pre-existing $\mathrm{T}$ cell exhaustion may be a negative predictive biomarker of response to checkpoint inhibition.

Many groups have attempted to immunophenotype patients with glioma to better understand the baseline immune microenvironment of these tumors. In this way, the response to therapy in patients can be better defined. Immune infiltration varies widely between different grades of glioma. ${ }^{88-90}$ TAMs have been found to be one of the predominant immune cells found in patients with GBM. ${ }^{91}{ }^{92}$ Tregs are also one of the predominant immune cell types in the immune microenvironment and may contribute to immune suppression..$^{93}$ The increase in Tregs may be attributable to the secretion of the chemokine CCL2 by glioma tumors, which induces Treg migration into the tumor microenvironment. ${ }^{95}$ Single cell analyses of myeloid cells in the GBM microenvironment revealed that there is a high degree of intertumor heterogeneity between different patients and intratumor heterogeneity in comparison to healthy brain of the same patient. ${ }^{96}$ One group recently evaluated the $\mathrm{T}$ cells of patients with glioma in comparison to healthy controls and found that GBM tumors cause infiltrating $\mathrm{T}$ cell exhaustion and dysfunction, in spite of presence of recruited T cells. ${ }^{97} \mathrm{~A}$ study led by Kimiecik et al assessed the relationship between the frequency of lymphocyte infiltration and OS of patients with glioma treated with chemotherapy and radiation. ${ }^{98}$ They noticed extended survival in these patients was associated with increased $\mathrm{CD}^{+}$and $\mathrm{CD}^{+}$immune cell infiltrates. However, the formal Immunoscore, as reported by Galon et al, has not been applied to GBM patient databases. Such investigations could show that the Immunoscore is an important prognostic variable in patients with glioma, leading to its inclusion in patient stratification for glioma clinical trials.

One ex vivo study of TILs isolated from GBM tissue demonstrated a high percentage of exhausted $\mathrm{T}$ cells that overexpress multiple checkpoint molecule receptors. ${ }^{99}$ This group found that ex vivo treatment of these TILs with checkpoint inhibitors caused a subpopulation of $\mathrm{T}$ cells with a less differentiated phenotype to be reinvigorated.

It is currently unclear how standard therapy impacts the GBM immune microenvironment. A recent study described the immune infiltration of gliomas using IHC and found no significant difference in primary and recurrent GBMs, but this study was limited by a low number of matched samples. ${ }^{100}$ Another study suggests that an increase in Tregs in the perivascular space correlates to a decreased time to recurrence following standard treatments. ${ }^{101}$
Such immune phenotyping has been applied to clinical trial biomarker investigations. A pilot study investigating autologous cancer cell vaccinations using anti-CD3stimulated lymphocytes in patients with recurrent grade III or IV astrocytoma found that the CD4/CD8 ratio of infused cells correlated with clinical outcomes, along with tumor grade and age. ${ }^{102}$ Additionally, patients with perivascular lymphocytic infiltration in their glioma tissue had a 4-month increase in survival compared with those with no lymphocyte infiltration.

Similarly, O'Rourke et al compared preinfusion and postinfusion EGFRvIII expression levels and immunosuppressive molecules in the tumor microenvironment while investigating peripherally infused EGFRvIII CAR T cell therapy for patients with recurrent GBM. ${ }^{103}$ They found a decrease in EGFRvIII expression in five of the seven patients who underwent postinfusion surgical resection and an increase in $\mathrm{FOXP}^{+}$cell frequency, IDO1, IL-10, PD-L1 and TGF- $\beta$. Interestingly, an increase in CD8 ${ }^{+}$ $\mathrm{T}$ cell proliferation was also noted in three of the five patients evaluated.

Researchers have also investigated how immune phenotyping of peripheral immune cells may yield useful biomarkers in the future. This technique is easily performed, relatively inexpensive and less invasive than GBM tissue sampling. A phase I study assessed the use of VXMO1, a plasmid containing an attenuated Salmonella typhi, TY21a that encodes vascular endothelial growth factor receptor-2 (VEGFR-2), in patients with progressive GBM. ${ }^{104}$ This vaccine recruits VEGFR-2-targeting $\mathrm{T}$ cells and stimulates a systemic immune response. They performed $\mathrm{T}$ cell immune monitoring in the peripheral blood and IHC on brain tissue and discovered that a higher CD8-to-Treg ratio was associated with increased survival in patients with primary tumors. This ratio was also increased by VXM01 treatment. Additionally, a decrease in intratumoral PD-L1 expression correlated with increased survival, suggesting the benefit of antiPD-L1 checkpoint inhibition in combination with VXM01.

A recent clinical trial performed a subset analysis of adjuvant intralesional autologous lymphokine-activated killer cell therapy for patients with primary GBM to differentiate responders versus non-responders. ${ }^{105}$ The researchers obtained autologous peripheral lymphokine-activated killer cells and incubated them in IL-2 prior to infusion into the tumor. They discovered that patients with more frequent $\mathrm{CD}^{+} \mathrm{CD} 16^{+} \mathrm{CD} 56^{+}$lymphokine-activated killer cells were associated with improved survival. Notably, this finding was more frequent in patients who did not receive corticosteroids in the month prior to leukapheresis.

A phase I clinical trial for recurrent malignant glioma evaluated the safety and efficacy of DNX-240, a tumorselective replication-competent oncolytic adenovirus, and measured pre-treatment and post-treatment levels of checkpoint proteins and immune cell density in biopsy tissue. $^{106}$ Comparing pre-treatment and post-treatment samples, they observed a decrease in TIM-3 levels 
following DNX-2401 administration, suggesting TIM-3 as a potential biomarker of response.

\section{Cytokine levels as biomarkers}

Beyond characterizing the presence of peripheral or tumor-infiltrating immune cells, measuring cytokine levels provides data regarding immune signaling and function. This may provide valuable insight into the complex relationship between pro-inflammatory and immunosuppressive signals in GBM and therapeutic response. Preclinical studies have suggested the potential utility of cytokine levels as biomarkers. For example, $\mathrm{Wu}$ et al found that GL261-Luc ${ }^{+}$-implanted mice treated with combination anti-PD-1 and anti-CXCR4 immunotherapy showed decreased production of pro-inflammatory cytokines including tumor necrosis factor- $\alpha$ and IFN- $\gamma$ compared with controls. ${ }^{107}$

Clinical trials have reported cytokines as potential biomarkers for GBM, but their significance and utility remain unclear. Inogés et al conducted a phase II study of autologous DC vaccination in newly diagnosed patients with GBM. ${ }^{108}$ They found an increase in tumor-specific immune cell response after vaccination in 11 out of the 27 patients by measuring proliferation and cytokine production. However, they did not see any correlation between this immune response and survival.

Nonetheless, cytokine measurement is being increasingly incorporated into prospective study design. A phase I clinical trial studying the safety of administering vaccine therapy made from survivin peptide in conjugation with sargramostim, a granulocyte macrophage colonystimulating factor (GM-CSF), for patients with GBM are defining treatment responders by measuring the IFN- $\gamma$ levels for up to 6 months post-treatment. ${ }^{109}$

\section{Tumor cell antigens as biomarkers}

Outside of immune-specific markers, many tumor cell surface molecules have been identified in many malignancies, including GBM. These tumor-specific antigens are useful as targets in vaccine development and can also give insight into treatment response. Sampson et al conducted a phase II multicenter clinical trial to determine the immunogenicity of an EGFRvIII vaccine for patients with newly diagnosed EGFRvIII ${ }^{+}$GBM. $^{110}$ They discovered that delayed hypersensitivity responses to EGFRvIII, as well as the development of specific antibodies to EGFRvIII, significantly affected OS. Additionally, they noted that $82 \%$ of the patients who had recurrence had lost EGFRvIII expression in their tumors. The phase III multicentre trial of rindopepiput, an EGFRvIII vaccine, with temozolomide (TMZ) in newly diagnosed GBM cases reported negative outcomes and attributed their results to the differences in EGFRvIII expression in their patient cohort. ${ }^{111}$ These findings conflicted with the positive improvement in median OS (mOS) following vaccine treatment in the preceding phase II clinical trials. ${ }^{112}$

Tumor antigens may also act as biomarkers even when those antigens are not specifically targeted. A phase
I clinical trial evaluating the immune response to a multiepitope-pulsed autologous dendritic cell vaccine in newly diagnosed patients with GBM showed that the expression of MAGE1 and AIM-2 on glioma cells was associated with significantly increased PFS. ${ }^{113}$ Additionally, they showed the expression levels of AIM-2 and MAGE1 correlated with both PFS and OS. For the five patients who underwent a second resection, there was a trend for increased survival with patients having tumors expressing gp100 and HER2 antigens, along with a decrease in CD133 expression levels.

\section{PD-L1 as a biomarker in glioblastoma}

PD-L1 expression has been widely investigated and reported as a potential biomarker within tumors and peripherally. An early analysis of PD-L1 expression on 135 specimens with GBM from both newly diagnosed and recurrent patients showed no clinical predictive or prognostic value. ${ }^{114}$ Contrary to these findings, Nduom et al found prognostic value for PD-L1, showing that high expression levels were associated with worse clinical outcomes. ${ }^{115}$ Furthermore, Pratt et al found varied PD-L1 expression, with high expression limited to a minority of patients. ${ }^{116}$

The clinical trial Checkmate 143 investigated the use of a PD-1 monoclonal antibody to improve median survival in recurrent patients with GBM in comparison to an antiVEGF antibody, bevacizumab. ${ }^{8117}$ They also retroactively measured PD-L1 expression to determine if the variability in expression could be used to distinguish responders from non-responders. However, this treatment did not increase mOS when compared with the control arm.

A phase II clinical trial using an autologous heat shock protein peptide vaccine, in combination with standard treatment, demonstrated decreased survival for newly diagnosed patients with GBM with high levels of PD-L1 on circulating myeloid cells, and elevated systemic immunosuppression. ${ }^{118}$ This association of PD-L1 expression and survival was not dependent on methylation status and was highly predictive of patient response to vaccine treatment.

\section{Genetic biomarkers}

Genetic and epigenetic aberrations are frequent in malignancy, including GBM. Further investigation of these features may reveal markers of response to immune therapy.

$\mathrm{O}^{6}$-methylguanine-DNA methyltransferase (MGMT) methylation is a predictive biomarker for response to standard chemoradiation. ${ }^{119}$ MGMT methylation status may play a similar role in immunotherapy. MGMTmethylation is associated with significantly improved survival compared with unmethylated MGMT patients in GBM vaccine therapy trials. ${ }^{12} 120$ Liau et al investigated autologous dendritic cell vaccine in newly diagnosed patients with GBM in a phase III clinical trial. ${ }^{121}$ They demonstrated that patients with a methylated MGMT had 
A.

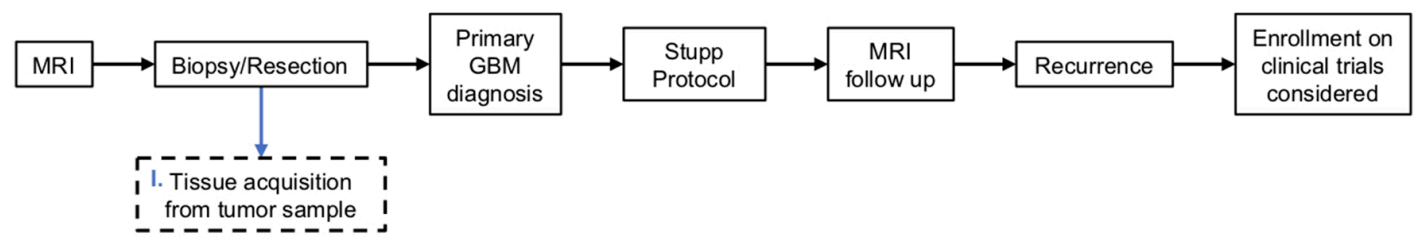

B.

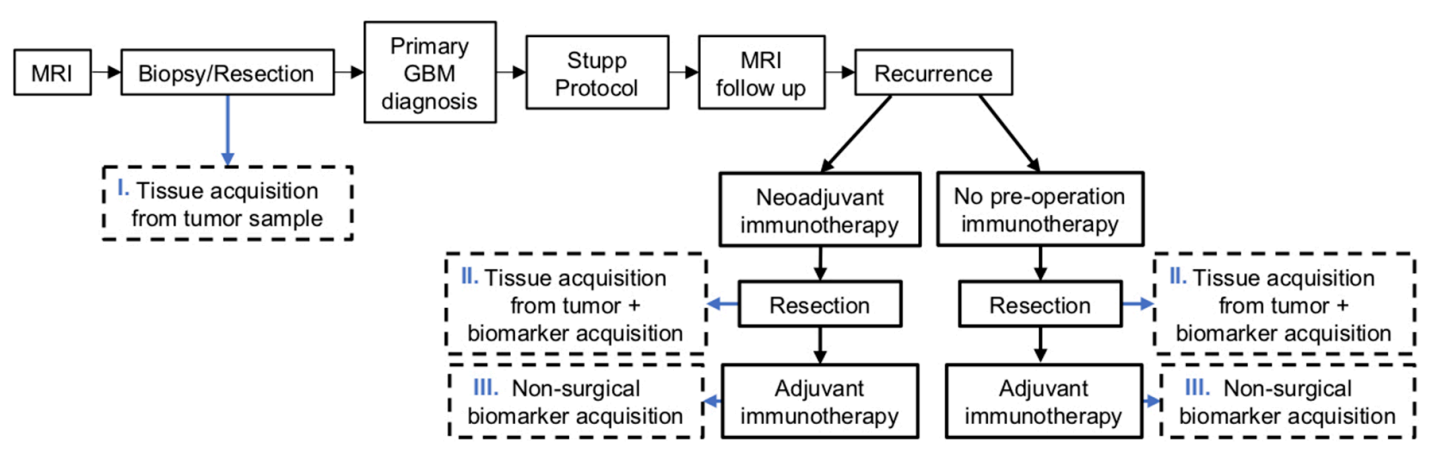

C.

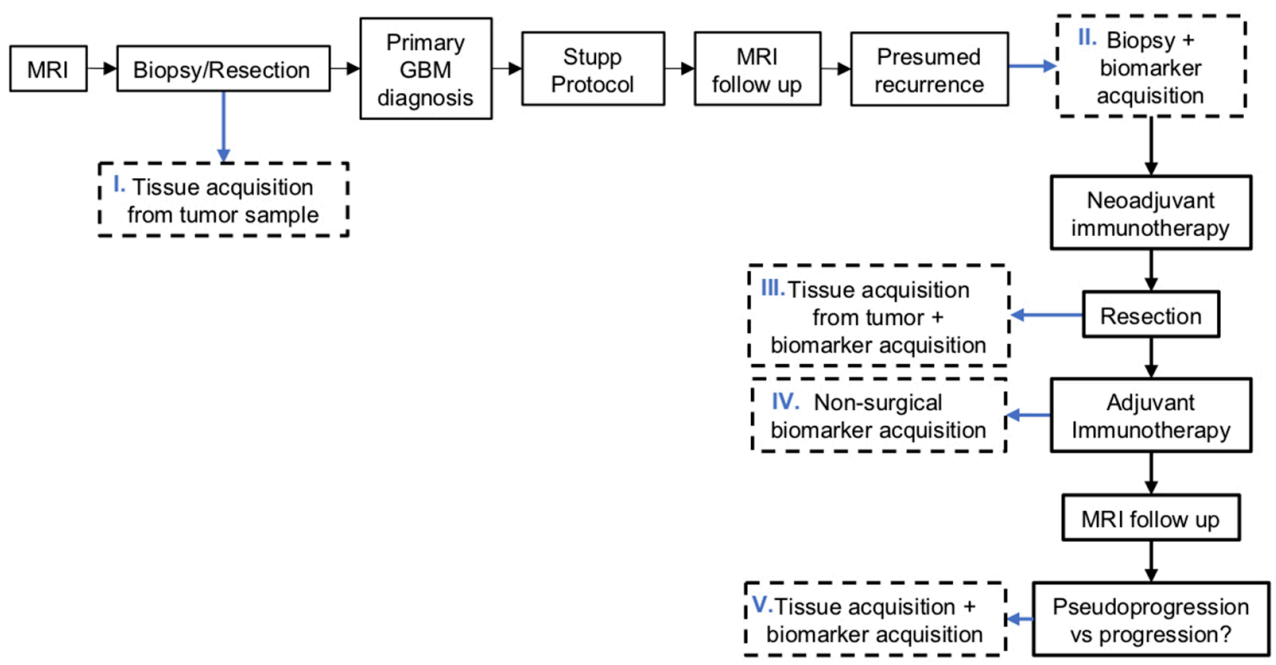

Figure 1 Different biomarker acquisition timepoints. (A) Standard. The current standard treatment course for patients with glioblastoma (GBM) used by practicing physicians. With this standard approach, biomarkers are only acquired from the primary resection tumor sample and usually without additional tissue samples taken during any subsequent trials. (B) Randomized neoadjuvant vs adjuvant immune therapy at recurrence. This schematic describes a more innovative approach to clinical trial design practiced by a few physicians allowing for more timepoints to acquire biomarkers. Following standard treatment, recurrence is suspected from MR imaging and patients can be enrolled on a trial to be treated with neoadjuvant immunotherapy prior to resection of the recurrent tumor or adjuvant immunotherapy after the second resection. Here, biomarkers can be collected from the primary tumor (I) and from recurrent tumor tissue following \pm neoadjuvant immunotherapy (II). Additionally, non-surgical biomarkers can be collected during follow-up adjuvant immunotherapy visits (III). (C) Proposed trial scheme to maximize biomarker identification. The proposed ideal approach to designing clinical trials to ensure physicians obtain biomarkers at all critical timepoints. First, biomarkers are collected from the primary tumor sample from the initial biopsy and/or resection (I). On suspected recurrence on MRI, a second biopsy will be performed to confirm recurrence vs pseudoprogression and to collect biomarkers of the recurrent tumor prior to any immunotherapy treatment (II). Third, biomarkers will be collected from resected tumor tissue after neoadjuvant administration of immunotherapy (III). Fourth, during adjuvant immunotherapy treatment, non-surgical biomarkers can be acquired with patient follow ups (IV). Lastly, should a patient present with progression vs pseudoprogression again, biomarkers can be collected during a therapeutic surgical intervention (V). 
A.

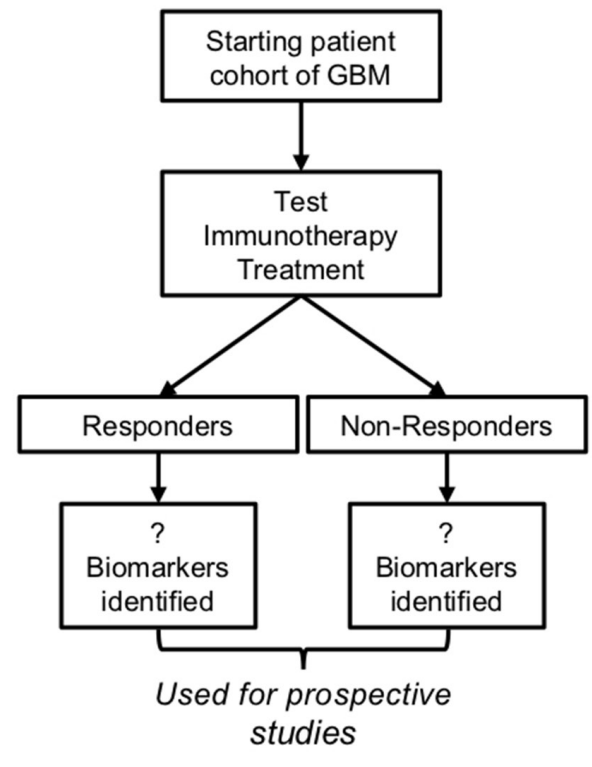

B.

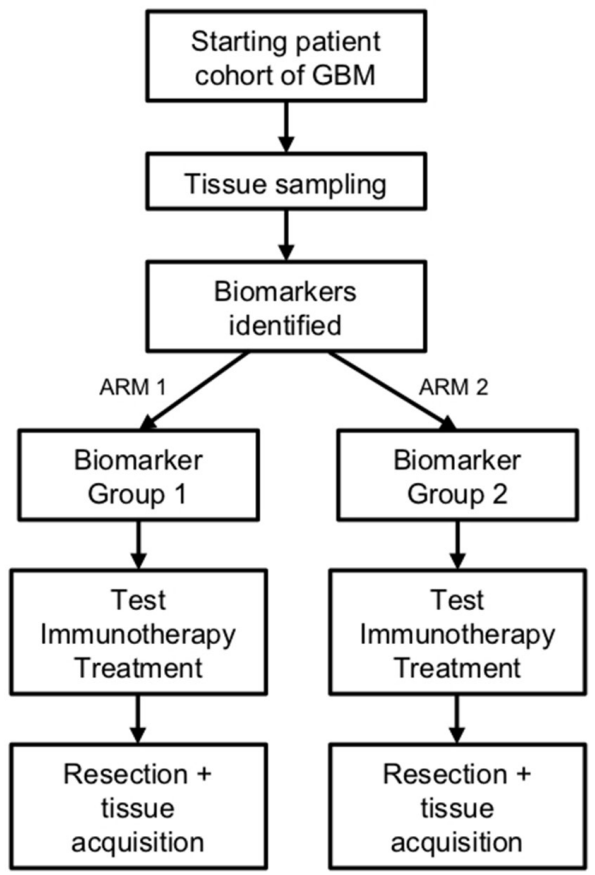

Figure 2 Immunotherapy trial design to find new predictive biomarkers. (A) A flow chart detailing how retrospective clinical trials can identify predictive biomarkers. As patients enroll in immunotherapy clinical trials, researchers can retrospectively delineate the responders to treatment from the non-responders and determine biomarker differences (mutation vs wild type, presence vs absence, high vs low expression). (B) Description of how identified predictive biomarkers in retrospective studies can be rigorously tested in prospective studies. On enrollment, patients will have their tissue sampled to identify changes in biomarkers identified in the cognate retrospective study. Then they will be stratified according to these criteria and administered treatment. Response from both groups will be compared to determine if the identified biomarkers are truly predictive of response to treatment.

a better mOS of 34.7 months compared with 21.2 months in historic controls.

Mutational status in the tumor suppressor gene, phosphatase and tensin homolog (PTEN), may also be a predictive biomarker. A study conducted by Zhao et al evaluated the immune response of anti-PD-1 treatment in patients with GBM using genomic and transcriptomic analyzes. ${ }^{122}$ They discovered that the non-responders had PTEN mutations associated with immunosuppression, while responders had enriched MAPK pathways.

Isocitrate dehydrogenase (IDH) mutational status has been demonstrated as a prognostic factor in primary $\mathrm{GBM},{ }^{123}$ but this may not be a predictive marker for immune therapy. Desjardins et al evaluated the use of recombinant poliovirus in recurrent patients with GBM. ${ }^{124}$ In a retrospective analysis of their patient population, they did not find a survival advantage for patients who harbored the IDH1 mutation.

Recently, advances in genomic sequencing have allowed for more comprehensive analysis of newly discovered genes found in tumors, including in GBM. These genes can potentially act as biomarkers in GBM and its treatment with immunotherapy. Sequencing has given insight into GBM mutagenesis and oncogenesis, with groups reporting many GBMs demonstrating DNA damage repair deficiencies, ${ }^{125}$ extensive cell-to-cell heterogeneity from chromosomal instability ${ }^{126}$ and marked intratumoral genetic heterogeneity. ${ }^{127-129}$

Peng et al used sequencing to identify markers of genetic instability in long-term survivors of GBM following standard treatment. ${ }^{130}$ This group suggested that patients who do not fall within this category may be ideal targets for non-standard therapies such as immune therapy. Feng et al identified a series of immune-related genes associated with GBM prognosis and found that the heterogeneity within the lymphocyte population is associated with a greater degree of immune infiltration into the tumor. ${ }^{131}$ This gene panel could serve as a predictive biomarker for response to immune therapy.

\section{Mutational burden}

The relationship of mutational burden with immune therapy response in GBM remains nebulous. One study reported dramatic responses to anti-PD-1 checkpoint inhibition in two patients with the DNA mismatch repair deficiency known as Lynch disease. ${ }^{132}$ However, relatively few GBMs have high mutational burden as compared with other malignancies. ${ }^{133}$

\section{Delayed-type hypersensitivity reactions}

Delayed-type hypersensitivity (DTH) skin reactions, a measure of an inflammatory response to a specific foreign 


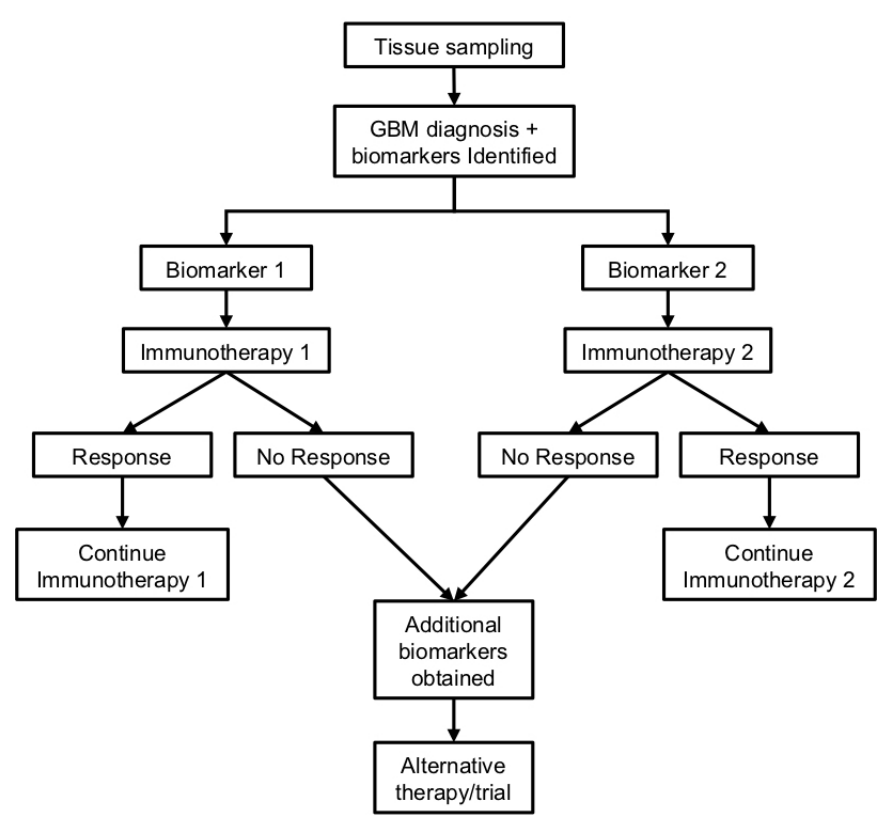

Figure 3 Trial scheme for the use of previously identified predictive biomarkers. A flow chart depicting how predictive biomarkers can be used in current clinical practices. When patients are initially diagnosed with glioblastoma (GBM), their tissue will be sampled to determine the presence of predictive biomarkers. Depending on the biomarkers present, patients will be enrolled in clinical trials that have shown response to treatment when that biomarker is present. If the patient is responding to treatment they will remain on the clinical trial, but if not, they can be switched to a different clinical trial to continue a different immune therapy treatment.

antigen, have also been used as a marker of immune response in clinical trials. ${ }^{134}$ A phase II multicenter trial exploring the immunogenicity and PFS of an EGFRvIII vaccine for newly diagnosed patients with GBM used patient serum and a DTH skin test with PEPvIII peptide to gauge the vaccine-induced immune response, ${ }^{135}$ elucidating additional clinical methods to identify minimally invasive biomarkers.

Similarly, a clinical trial conducted by Schneider et al used tumor cells modified with Newcastle Disease Virus (NDV) for 11 patients with GBM. ${ }^{136}$ Autologous tumor cells were extracted from the patients and incubated with NDV, then re-injected subcutaneously. They postulated that the vaccine would stimulate the immune system and show a local inflammatory response in skin with a corresponding relationship between OS and the rate of onset of the reaction. There was no significant correlation between area of skin inflammation and rate of systemic response or OS. However, the tumor samples of patients who underwent a second surgery after immunotherapy demonstrated a higher $\mathrm{CD} 4^{+} / \mathrm{CD}^{+} \mathrm{T}$ cell infiltrating ratio following immunotherapy.

\section{Multimodal biomarkers}

Due to the complexity of the immune system in the disease state and in response to treatment, the simultaneous use of multiple biomarkers may best inform researchers in trial design and subsequent analysis. Additionally, it allows for more data and potential discovery to occur from fewer studies.

The ongoing phase I/II clinical trial for the IMA950 multipeptide vaccine in combination with poly-ICLC for GBM is seeking to determine if there is a correlation between clinical response and immunological response. ${ }^{137}$ They sample blood to measure cytokine secretion and proliferation of antigen-specific $\mathrm{CD}^{+}$ and $\mathrm{CD} 4^{+} \mathrm{T}$ cells, frequency of myeloid cells and Tregs, and activation marker expression on tetramer positive cells. This study may show effective-response monitoring using only peripheral blood samples, benefiting patients and clinicians due to its relative non-invasiveness and ease of future implementation.

The randomized multi-institutional clinical trial conducted by the Ivy Foundation Early Phase Clinical Trials Consortium used gene analysis when aiming to demarcate immune therapy responders from nonresponders. ${ }^{138}$ The study examined the immune response of 35 recurrent patients with GBM receiving neoadjuvant and/or adjuvant anti-PD-1 therapy. They noted that the use of neoadjuvant anti-PD-1 correlated with an upregulation of IFN- $\gamma$-related gene expression, while downregulating cell cycle-related genes in the tumor when compared with the adjuvant anti-PD-1 group. Additionally, they showed that this neoadjuvant treatment increased clonal $\mathrm{T}$ cell expansion, attenuated PD-L1 expression on peripheral blood T cells and decreased monocyte frequency. Neoadjuvant anti-PD-1 immune response was also characterized in a phase II single-arm study that compared pretumor and post-tumor tissue samples after treatment. ${ }^{139}$ They showed that neoadjuvant anti-PD-1 increased immune cell infiltration, $\mathrm{T}$ cell receptor clonal diversity among infiltrating $\mathrm{T}$ cells and enhanced expression of chemokine transcripts.

\section{FUTURE DIRECTIONS FOR BIOMARKER IDENTIFICATION AND QUANTIFICATION}

Given the need for rigorous biomarker collection in future clinical trials, novel techniques of biomarker quantification are vital. Despite the current standard of treatment for patients with GBM (figure 1A), biomarker collection should rely on repeat tissue sampling (figure 1B,C), as immunotherapy efficacy is difficult to interpret using MR imaging alone. ${ }^{140}$ Stereotactic biopsy has a low side-effect profile and provides pathological diagnosis and potential biomarkers that may be critical for effective stratification of patients to particular existing therapy or enrollment in a variety of active clinical trials (figure 2). While future trials may obviate the need for such tissue collection, we believe it is critical that tissue sampling and window of opportunity trials continue in patients with GBM until such less invasive biomarkers are developed (figure 3). 


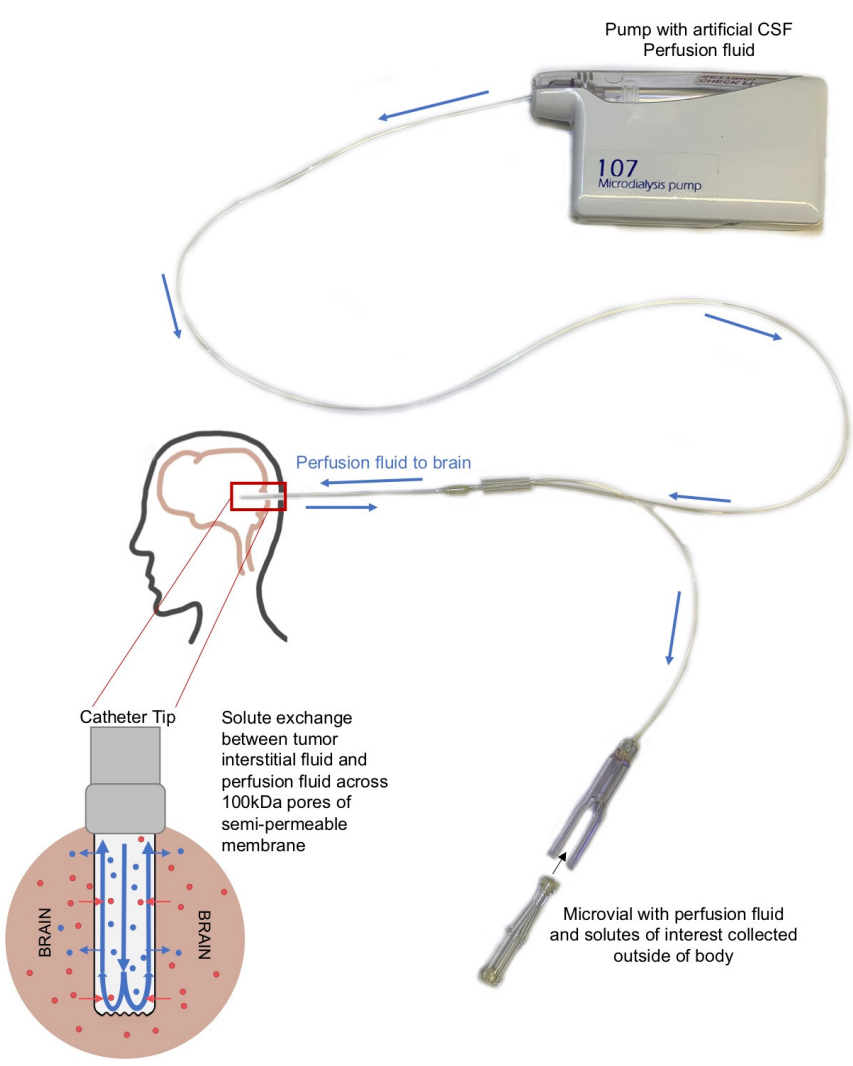

Figure 4 Cytokine microdialysis catheter mechanism. A diagram describing the mechanism of the microdialysis catheter. Artificial cerebrospinal fluid (CSF) perfusion fluid is pushed through the catheter using a microdialysis pump. When the fluid reaches the catheter tip, solutes are exchanged between the artificial CSF perfusion fluid and the interstitial fluid of the brain through a semi-permeable membrane. The resulting mixture is then pushed into a microvial where it can be collected and analyzed.

\section{Clinical trial design}

Moving forward, there are many ways biomarkers can be used to improve immunotherapy for patients with GBM. Clinical trials can be designed both retrospectively and prospectively to look at responders and non-responders to immunotherapy. By stratifying patients by response, researchers can identify which biomarkers can be used to differentiate these two groups (figure 2A). Subsequent trials can determine if these biomarkers have predictive value by prospectively stratifying their patient cohort based on the biomarkers and comparing their treatment response with controls (figure 2B). This allows systematic, targeted immunotherapy for patients with GBM that will most likely benefit from a particular treatment regimen (figure 3).

\section{Microdialysis catheters}

Cerebral microdialysis samples interstitial fluid of the brain using catheter placement ${ }^{141}$ (figure 4 ). This technology has been demonstrated to be safe to patients and is able to measure immune biomarkers in the context of cerebrovascular injury ${ }^{142}$ and traumatic brain injury. ${ }^{143}$ The use of microdialysis catheters for biomarker acquisition can provide clinicians with direct access to the tumor microenvironment by sampling the interstitial fluid of the brain and/or tumor. This may obviate the concern that biomarkers of response collected from other fluid compartments of the body, such as the blood or cerebrospinal fluid (CSF), may be diluted as compared with the interstitial fluid. ${ }^{144-146}$ Small early phase trials demonstrated the potential utility of cerebral microdialysis for cytokines in neuro-oncology. Portnow et al reported changes in 17 cytokines following chemotherapy for various intracerebral malignancies including GBM via microdialysis monitoring over 96 hours following craniotomy. ${ }^{147}$ Similarly, this technique has demonstrated immune response in the GBM peritumoral region following radiation therapy. ${ }^{148}$ The ongoing study 'Cytokine Microdialysis for Immune Monitoring in Recurrent Glioblastoma Patients Undergoing Checkpoint Blockade' uses microdialysis catheters to sample the tumor and normal brain microenvironments while concurrently collecting peripheral blood mononuclear cells and CSF of patients undergoing neoadjuvant antiPD-1 immunotherapy prior to secondary surgical resection with continued infusions of combination of anti-PD-1 and anti-LAG-3 immunotherapy postoperatively. ${ }^{149}$ This comprehensive approach may identify new biomarkers for response, or lack thereof, in checkpoint inhibition for GBM as well as correlate between markers in different physiological spaces (figure 1C).

\section{Decreased invasive sampling}

Recently, less invasive techniques to collect biomarkers to monitor response to treatment have been explored. Liquid biopsies have also been proposed as an alternative to conventional tissue biopsies in evaluation of treatment response. ${ }^{150}$ These biopsies typically involve the use of peripheral blood to identify the presence of intact circulating tumor cells, circulating tumor DNA (ctDNA) and other tumor-derived molecules that have entered into the bloodstream or CSF from primary or secondary tumors. ${ }^{127}$ Since liquid biopsies examine cancer-related factors present in the peripheral blood or CSF, they provide clinicians with a less invasive approach than typical tissue biopsies to acquire biomarkers. Potential biomarkers, including ctDNA, peripheral blood mononuclear cells and microRNA, have been found to be differentially present within serum, urine, stool and/or saliva samples after immune therapy. ${ }^{151}$ Kitahara et al used plasma ctDNA levels to monitor response to a peptide vaccine in patients with colorectal cancer. ${ }^{152}$ Cabel et al used ctDNA to distinguish tumor progression from pseudoprogression in patients with melanoma following checkpoint inhibition therapy and found that loss of detectable ctDNA at 8 weeks after treatment correlated with durable clinical response and improvement survival. ${ }^{153}$ Another study of 200 patients with breast, lung, colorectal or ovarian cancer showed a high concordance of mutations in ctDNA and tumor tissue. ${ }^{154}$ Additionally, an analysis of the colorectal 
patient cohort revealed that high levels of ctDNA was associated with lower OS and disease recurrence.

Interestingly, microbiota within stools samples have also been shown to play a potential role in immune therapy response. Matson et al analyzed baseline stool samples from patients with multiple melanoma before immunotherapy treatment and observed a significant correlation between commensal microbial composition and clinical response, finding Bifidobacterium longum, Collinsella aerofaciens and Enterococcus faecium to be more abundant in responders. ${ }^{155}$ Overall, although this field is relatively new, non-invasive strategies for biomarker collection may prove to be beneficial in stratifying patients and treatmentresponse monitoring for future immune therapy trials.

\section{Non-invasive imaging development}

Discerning pseudoprogression from progression of the disease based on imaging is a notoriously difficult problem because the inflammatory response can often mimic non-enhancing tumor progression. ${ }^{140}$ New imaging modalities are required that can either more accurately detect immune infiltration or specifically identify tumor progression separate from inflammation.

Antonios et al developed a non-invasive combination imaging technique to differentiate immune inflammatory changes from tumor progression in intracranial murine gliomas and in patients with GBM after dendritic cell vaccination and/or PD-1 inhibition. ${ }^{156}$ Their combination of MRI and PET imaging with a probe for deoxycytidine kinase (dCK), a protein marker overexpressed in immune cells, is a new technique that was able to effectively image immune response in intracranial tumors in preclinical murine models and in patients. Similarly, Rashidian et al use PET imaging to determine infiltration of $\mathrm{CD}^{+}, \mathrm{CD} 11 \mathrm{~b}^{+}$and $\mathrm{CD} 45^{+}$immune cells in tumors in response to anti-PD-1 treatment in a murine model. ${ }^{157}$ These modalities may provide clinicians a non-invasive way to monitor response of immunotherapy in GBM tumors, a critical necessity in measuring efficacy in future clinical trials.

\section{CONCLUSION}

As immune therapy continues to expand in response to promising results for malignancies with poor prognosis, the demand for effective biomarkers will only continue to increase. The discovery and development of effective techniques to predict therapeutic efficacy and measure patient response have the potential to significantly improve clinical decision-making. By enabling clinicians to treat patients with immune therapeutics that have the highest chance of evoking an objective response, biomarker development will improve the practice of immune therapeutics. The future of biomarker development will maximize positive outcomes to patients while minimizing exposure to ineffective therapy with potential risk.

\section{Twitter Edjah K Nduom @eknduom}

Contributors JL, AN, HS, EKN conceptualized, wrote, designed the figures, and edited the paper. VS, KS, OA, GD conceptualized, wrote and edited the paper. All authors read and approved the final manuscript.

Funding This research was supported by the Intramural Research Program of the NINDS at the National Institutes of Health.

Competing interests None declared.

Patient consent for publication Not required.

Provenance and peer review Not commissioned; externally peer reviewed.

Open access This is an open access article distributed in accordance with the Creative Commons Attribution Non Commercial (CC BY-NC 4.0) license, which permits others to distribute, remix, adapt, build upon this work non-commercially, and license their derivative works on different terms, provided the original work is properly cited, appropriate credit is given, any changes made indicated, and the use is non-commercial. See http://creativecommons.org/licenses/by-nc/4.0/.

\section{REFERENCES}

1 Lynes J, Sanchez V, Dominah G, et al. Current options and future directions in immune therapy for glioblastoma. Front Oncol 2018;8:578.

2 Larkin J, Chiarion-Sileni V, Gonzalez R, et al. Combined nivolumab and ipilimumab or monotherapy in untreated melanoma. $N$ Engl $J$ Med Overseas Ed 2015;373:23-34.

3 Garon EB, Rizvi NA, Hui R, et al. Pembrolizumab for the treatment of Non-Small-Cell lung cancer. N Engl J Med 2015;372:2018-28.

4 Sanders S, Stewart DA. Targeting non-Hodgkin lymphoma with blinatumomab. Expert Opin Biol Ther 2017;17:1013-7.

5 Enblad G, Karlsson H, Loskog ASI. Car T-cell therapy: the role of physical barriers and immunosuppression in lymphoma. Hum Gene Ther 2015;26:498-505.

6 Biomarkers Definitions Working Group. Biomarkers and surrogate endpoints: preferred definitions and conceptual framework. Clin Pharmacol Ther 2001;69:89-95.

7 Dobbin KK, Cesano A, Alvarez J, et al. Validation of biomarkers to predict response to immunotherapy in cancer: volume II - clinical validation and regulatory considerations. J Immunother Cancer 2016;4:77.

8 Squibb B-M. A study of the effectiveness and safety of nivolumab compared to bevacizumab and of nivolumab with or without ipilimumab in glioblastoma patients (CheckMate 143), 2019. Available: https://clinicaltrials.gov/ct2/show/NCT02017717

9 Squibb B-M. An investigational Immuno-therapy study of nivolumab compared to temozolomide, each given with radiation therapy, for Newly-diagnosed patients with glioblastoma (GBM, a malignant brain cancer) (CheckMate 498). Available: https://clinicaltrials.gov/ ct2/show/NCT02617589

10 Squibb B-M. An investigational Immuno-therapy study of temozolomide plus radiation therapy with nivolumab or placebo, for newly diagnosed patients with glioblastoma (GBM, a malignant brain cancer) (CheckMate548), 2019. Available: https://clinicaltrials. gov/ct2/show/NCT02667587

11 Gedeon PC, Riccione KA, Fecci PE, et al. AntibodyBased immunotherapy for malignant glioma. Semin Oncol 2014;41:496-510.

12 Chinot OL, Wick W, Mason W, et al. Bevacizumab plus Radiotherapy-Temozolomide for newly diagnosed glioblastoma. $N$ Engl J Med Overseas Ed 2014;370:709-22.

13 Ostrom QT, Gittleman H, Fulop J, et al. CBTRUS statistical report: primary brain and central nervous system tumors diagnosed in the United States in 2008-2012. Neuro Oncol 2015;17:iv1-62.

14 Nduom EK, Weller M, Heimberger AB. Immunosuppressive mechanisms in glioblastoma: fig. 1. Neuro Oncol 2015;17:vii9-14.

15 Kleihues P, Louis DN, Scheithauer BW, et al. The who classification of tumors of the nervous system. J Neuropathol Exp Neurol 2002;61:215-25.

16 Shapiro JR, Yung WK, Shapiro WR, Isolation SWR. Isolation, karyotype, and clonal growth of heterogeneous subpopulations of human malignant gliomas. Cancer Res 1981;41:2349-59.

17 Wikstrand CJ, Bigner SH, Bigner DD. Demonstration of complex antigenic heterogeneity in a human glioma cell line and eight derived clones by specific monoclonal antibodies. Cancer Res 1983;43:3327-34.

18 Jung V, Romeike BFM, Henn W, et al. Evidence of focal genetic microheterogeneity in glioblastoma multiforme by area-specific 
CGH on microdissected tumor cells. J Neuropathol Exp Neurol 1999;58:993-9.

19 Chen P-L, Roh W, Reuben A, et al. Analysis of immune signatures in longitudinal tumor samples yields insight into biomarkers of response and mechanisms of resistance to immune checkpoint blockade. Cancer Discov 2016;6:827-37.

20 Yoshida J, Ishikawa T, Doi T, et al. Clinical significance of soluble forms of immune checkpoint molecules in advanced esophageal cancer. Med Oncol 2019;36:60.

21 Shen K, Cui J, Wei Y, et al. Effectiveness and safety of PD-1/ PD-L1 or CTLA4 inhibitors combined with chemotherapy as a first-line treatment for lung cancer: a meta-analysis. J Thorac Dis 2018;10:6636-52.

$22 \mathrm{Liu} \mathrm{C,} \mathrm{He} \mathrm{H,} \mathrm{Li} \mathrm{X,} \mathrm{et} \mathrm{al.} \mathrm{Dynamic} \mathrm{metrics-based} \mathrm{biomarkers} \mathrm{to}$ predict responders to anti-PD-1 immunotherapy. $\mathrm{Br} \mathrm{J}$ Cancer 2019;120:346-55

23 Chaft JE, Hellmann MD, Velez MJ, et al. Initial experience with lung cancer resection after treatment with T-cell checkpoint inhibitors. Ann Thorac Surg 2017;104:e217-8.

24 Schatton T, Scolyer RA, Thompson JF, et al. Tumor-Infiltrating lymphocytes and their significance in melanoma prognosis. Methods Mol Biol 2014;1102:287-324.

$25 \mathrm{Lu}$ J, Xu Y, Wu Y, et al. Tumor-Infiltrating CD8 ${ }^{+} \mathrm{T}$ cells combined with tumor-associated $\mathrm{CD} 8^{+}$macrophages predict postoperative prognosis and adjuvant chemotherapy benefit in resected gastric cancer. BMC Cancer 2019;19:920.

26 Park JH, Jonas SF, Dieci MV, Bataillon G, et al. Prognostic value of tumour infiltrating lymphocytes (TILs) in patients with earlystage triple negative breast cancers (TNBC) in the absence of chemotherapy. Annals of Oncology 2019;30:v55.

27 Shaban M, Khurram SA, Fraz MM, et al. A novel digital score for abundance of tumour infiltrating lymphocytes predicts disease free survival in oral squamous cell carcinoma. Sci Rep 2019;9:13341.

28 Honkanen TJ, Tikkanen A, Karihtala P, et al. Prognostic and predictive role of tumour-associated macrophages in HER2 positive breast cancer. Sci Rep 2019;9:10961.

29 Sanchez V, Lynes J, Dominah G, et al. TMOD-05. GLIOMA-261 LUCIFERASE-EXPRESSING cell line stimulates an immunogenic response signature in an immunocompetent murine model. Neuro Oncol 2018:20:269.

30 Djenidi F, Adam J, Goubar A, et al. CD8 ${ }^{+} \mathrm{CD}_{103^{+}}$tumor-infiltrating lymphocytes are tumor-specific tissue-resident memory $T$ cells and a prognostic factor for survival in lung cancer patients. J Immunol 2015;194:3475-86.

31 Komdeur FL, Prins TM, van de Wall S, et al. $\mathrm{CD}_{103^{+}}$tumorinfiltrating lymphocytes are tumor-reactive intraepithelial $\mathrm{CD}^{+} \mathrm{T}$ cells associated with prognostic benefit and therapy response in cervical cancer. Oncoimmunology 2017;6:e1338230.

32 Ou Y, Cannon MJ, Nakagawa M. Regulatory T cells in gynecologic cancer. MOJ Immunology 2018;6:34-42.

33 Gonzaga AKG, Santos HBdeP, Crispim JCO, et al. Immunohistochemical evaluation of HLA-G and Foxp3 $3^{+}$T regulatory cells in oral cavity and lower lip squamous cell carcinomas. Braz Oral Res 2019;33:e020.

34 Watanabe M, Kanao K, Suzuki S, et al. Increased infiltration of CCR4-positive regulatory T cells in prostate cancer tissue is associated with a poor prognosis. Prostate 2019;79:1658-65.

35 Peng G-L, Li L, Guo Y-W, et al. CD8 ${ }^{+}$cytotoxic and FoxP3 regulatory $\mathrm{T}$ lymphocytes serve as prognostic factors in breast cancer. Am J Transl Res 2019;11:5039-53.

36 Zhu G, Pei L, Yin H, et al. Profiles of tumor-infiltrating immune cells in renal cell carcinoma and their clinical implications. Oncol Lett 2019;18:5235-42.

37 Daayana S, Elkord E, Winters U, et al. Phase II trial of imiquimod and HPV therapeutic vaccination in patients with vulval intraepithelial neoplasia. Br J Cancer 2010;102:1129-36.

38 Stanley MA. Imiquimod and the imidazoquinolones: mechanism of action and therapeutic potential. Clin Exp Dermatol 2002;27:571-7.

39 Abiko K, Matsumura N, Hamanishi J, et al. IFN- $\gamma$ from lymphocytes induces PD-L1 expression and promotes progression of ovarian cancer. Br J Cancer 2015;112:1501-9.

40 Schmid P, Cruz C, Braiteh FS, et al. Abstract 2986: Atezolizumab in metastatic TNBC (mTNBC): long-term clinical outcomes and biomarker analyses. Cancer Res 2017:77:2986.

41 Dirix LY, Takacs I, Jerusalem G, et al. Avelumab, an anti-PD-L1 antibody, in patients with locally advanced or metastatic breast cancer: a phase 1B javelin solid tumor study. Breast Cancer Res Treat 2018;167:671-86.

42 Forde PM, Chaft JE, Smith KN, et al. Neoadjuvant PD-1 blockade in resectable lung cancer. N Engl J Med 2018;378:1976-86.
43 Amaria RN, Reddy SM, Tawbi HA, et al. Neoadjuvant immune checkpoint blockade in high-risk resectable melanoma. Nat Med 2018;24:1649-54.

44 Sharma P, Retz M, Siefker-Radtke A, et al. Nivolumab in metastatic urothelial carcinoma after platinum therapy (CheckMate 275): a multicentre, single-arm, phase 2 trial. Lancet Oncol 2017:18:312-22.

45 Brahmer J, Reckamp KL, Baas P, et al. Nivolumab versus docetaxe in advanced squamous-cell non-small-cell lung cancer. $N$ Engl $J$ Med 2015:373:123-35.

46 Das R, Verma R, Sznol M, et al. Combination therapy with AntiCTLA-4 and Anti-PD-1 leads to distinct immunologic changes in vivo. J.i. 2015;194:950-9.

47 Tolcher AW, Sznol M, Hu-Lieskovan S, et al. Phase lb study of Utomilumab (PF-05082566), a 4-1BB/CD137 agonist, in combination with pembrolizumab (MK-3475) in patients with advanced solid tumors. Clin Cancer Res 2017;23:5349-57.

48 Ngoi NYL, Heong V, Lee XW, et al. Tumor molecular profiling of responders and non-responders following pembrolizumab monotherapy in chemotherapy resistant advanced cervical cancer. Gynecologic Oncology Reports 2018;24:1-5.

49 Fujiyama T, Oze I, Yagi $\mathrm{H}$, et al. Induction of cytotoxic $\mathrm{T}$ cells as a novel independent survival factor in malignant melanoma with percutaneous peptide immunization. J Dermatol Sci 2014;75:43-8.

50 Ayers M, Lunceford J, Nebozhyn M, et al. IFN- $\gamma$-related mRNA profile predicts clinical response to PD-1 blockade. J Clin Invest 2017;127:2930-40.

51 Gao J, Shi LZ, Zhao H, et al. Loss of IFN- $\gamma$ pathway genes in tumor cells as a mechanism of resistance to anti-CTLA-4 therapy. Cell 2016;167:397-404.

52 Klebanov N, Artomov M, Goggins WB, et al. Burden of unique and low prevalence somatic mutations correlates with cancer survival. Sci Rep 2019;9:4848.

53 Wang L, Gong Y, Saci A, et al. Fibroblast growth factor receptor 3 alterations and response to PD-1/PD-L1 blockade in patients with metastatic urothelial cancer. Eur Urol 2019;76:599-603.

54 Zang Y-S, Dai C, Xu X, et al. Comprehensive analysis of potential immunotherapy genomic biomarkers in 1000 Chinese patients with cancer. Cancer Med 2019;8:4699-708.

55 Alborelli I, Leonards K, Rothschild SI, et al. Tumor mutational burden assessed by targeted NGS predicts clinical benefit from immune checkpoint inhibitors in non-small cell lung cancer. $J$ Pathol 2020;250:19-29.

56 Heeke S, Benzaquen J, Long-Mira E, et al. In-House implementation of tumor mutational burden testing to predict durable clinical benefit in non-small cell lung cancer and melanoma patients. Cancers 2019;11:1271.

57 Rizvi H, Sanchez-Vega F, La K, et al. Molecular Determinants of Response to Anti-Programmed Cell Death (PD)-1 and AntiProgrammed Death-Ligand 1 (PD-L1) Blockade in Patients With Non-Small-Cell Lung Cancer Profiled With Targeted NextGeneration Sequencing. J Clin Oncol 2018;36:633-41.

58 Goodman AM, Kato S, Bazhenova L, et al. Tumor mutational burden as an independent predictor of response to immunotherapy in diverse cancers. Mol Cancer Ther 2017;16:2598-608.

59 Fakih M, Sandhu JS, Ouyang C, et al. Tumor mutational burden (TMB) may be a promising predictive biomarker of response to PD-1/PD-L1 targeting in MSI-H colorectal cancer. JCO 2019;37:43.

60 Redig AJ, Jänne PA. Basket trials and the evolution of clinical trial design in an era of genomic medicine. J Clin Oncol 2015;33:975-7.

61 Chau NG, Lorch JH. Exceptional responders Inspire change: lessons for drug development from the bedside to the bench and back. Oncologist 2015;20:699-701.

62 Chen $\mathrm{H}$, Chong W, Wu Q, et al. Association of LRP1B mutation with tumor mutation burden and outcomes in melanoma and nonsmall cell lung cancer patients treated with immune Check-Point blockades. Front Immunol 2019;10:1113.

63 Galon J, Costes A, Sanchez-Cabo F, et al. Type, density, and location of immune cells within human colorectal tumors predict clinical outcome. Science 2006;313:1960-4.

64 O'Sullivan B, Brierley J, Byrd D, et al. The TNM classification of malignant tumours-towards common understanding and reasonable expectations. Lancet Oncol 2017;18:849-51.

65 Galon J, Mlecnik B, Bindea G, et al. Towards the introduction of the 'Immunoscore' in the classification of malignant tumours. J Pathol 2014;232:199-209.

66 Galon J, Pagès F, Marincola FM, et al. The immune score as a new possible approach for the classification of cancer. J Transl Med 2012;10:1. 
67 Chaput N, Svrcek M, Aupérin A, et al. Tumour-Infiltrating $\mathrm{CD}^{+} 8^{+}$and $\mathrm{CD} 57^{+}$cells predict patient outcome in stage II-III colorectal cancer. Br J Cancer 2013;109:1013-22.

68 Pagès F, Kirilovsky A, Mlecnik B, et al. In situ cytotoxic and memory $T$ cells predict outcome in patients with early-stage colorectal cancer. J Clin Oncol 2009;27:5944-51.

69 Mlecnik B, Bindea G, Angell HK, et al. Integrative analyses of colorectal cancer show immunoscore is a stronger predictor of patient survival than microsatellite instability. Immunity 2016:44:698-711

70 Mlecnik B, Bindea G, Kirilovsky A, et al. The tumor microenvironment and immunoscore are critical determinants of dissemination to distant metastasis. Sci Trans/ Med 2016;8:327ra2 6-ra26.

71 Jiang $\mathrm{Y}$, Zhang Q, Hu Y, et al. Immunoscore signature: a prognostic and predictive tool in gastric cancer. Ann Surg 2018;267:504-13.

72 Galon J, Fox BA, Bifulco CB, et al. Immunoscore and Immunoprofiling in cancer: an update from the melanoma and immunotherapy bridge 2015. J Transl Med 2016;14:273.

73 Sun C, Xu J, Song J, et al. The predictive value of centre tumour $\mathrm{CD}^{+} \mathrm{T}$ cells in patients with hepatocellular carcinoma: comparison with immunoscore. Oncotarget 2015;6:35602-15.

74 Bösmüller H-C, Wagner $\mathrm{P}$, Peper JK, et al. Combined immunoscore of CD103 and CD3 identifies long-term survivors in high-grade serous ovarian cancer. Int J Gynecol Cancer 2016;26:671-9.

75 Bifulco C, Capone M, Feng Z, et al. MISIPI study: melanoma immunoscore evaluation in patients treated with ipilimumab. $J$ Transl Med 2014;12:P11.

76 McLemore LE, Janakiram M, Albanese J, et al. An immunoscore using PD-L1, CD68, and tumor-infiltrating lymphocytes (TILs) to predict response to neoadjuvant chemotherapy in invasive breast cancer. Appl Immunohistochem Mol Morphol 2018;26:611-9.

77 Hodi FS, O'Day SJ, McDermott DF, et al. Improved survival with ipilimumab in patients with metastatic melanoma. N Engl J Med 2010;363:711-23

78 Wolchok JD, Neyns B, Linette G, et al. Ipilimumab monotherapy in patients with pretreated advanced melanoma: a randomised, double-blind, multicentre, phase 2, dose-ranging study. Lancet Oncol 2010;11:155-64.

79 Tawbi HA, Forsyth PA, Algazi A, et al. Combined nivolumab and ipilimumab in melanoma metastatic to the brain. $N$ Engl $J$ Med 2018:379:722-30.

80 Capone M, Giannarelli D, Mallardo D, et al. Baseline neutrophilto-lymphocyte ratio (NLR) and derived NLR could predict overall survival in patients with advanced melanoma treated with nivolumab. J Immunother Cancer 2018;6:74.

81 Berghoff AS, Fuchs E, Ricken G, et al. Density of tumor-infiltrating lymphocytes correlates with extent of brain edema and overall survival time in patients with brain metastases. Oncoimmunology 2016;5:e1057388.

82 Wolchok JD, Hoos A, O'Day S, et al. Guidelines for the evaluation of immune therapy activity in solid tumors: immune-related response criteria. Clin Cancer Res 2009;15:7412-20.

83 Ranjan S, Quezado M, Garren N, et al. Clinical decision making in the era of immunotherapy for high grade-glioma: report of four cases. BMC Cancer 2018;18:239.

84 Okada H, Pollack IF. Do we need novel radiologic response criteria for brain tumor immunotherapy? Expert Rev Neurother 2011:11:619-22.

85 Zeng J, See AP, Phallen J, et al. Anti-Pd-1 blockade and stereotactic radiation produce long-term survival in mice with intracranial gliomas. Int J Radiat Oncol Biol Phys 2013;86:343-9.

86 Fecci PE, Ochiai H, Mitchell DA, et al. Systemic CTLA-4 blockade ameliorates glioma-induced changes to the $\mathrm{CD} 4^{+} \mathrm{T}$ cell compartment without affecting regulatory T-cell function. Clin Cancer Res 2007:13:2158-67.

87 Karachi A, Yang C, Dastmalchi F, et al. Modulation of temozolomide dose differentially affects T-cell response to immune checkpoint inhibition. Neuro Oncol 2019;21:730-41.

88 Han S, Zhang C, Li Q, et al. Tumour-Infiltrating CD4 ${ }^{+}$and CD8 lymphocytes as predictors of clinical outcome in glioma. $\mathrm{Br} \mathrm{J}$ Cancer 2014:110:2560-8.

89 Moyes KW, Davis A, Hoglund V, et al. Effects of tumor grade and dexamethasone on myeloid cells in patients with glioma. Oncoimmunology 2018;7:e1507668.

90 Gao Y, Weenink B, van den Bent MJ, et al. Expression-Based intrinsic glioma subtypes are prognostic in low-grade gliomas of the EORTC22033-26033 clinical trial. Eur J Cancer 2018;94:168-78.

91 Vidyarthi A, Agnihotri T, Khan N, et al. Predominance of M2 macrophages in gliomas leads to the suppression of local and systemic immunity. Cancer Immunol Immunother 2019;68:1995-2004.

92 Locarno CV, Simonelli M, Carenza C, et al. Role of myeloid cells in the immunosuppressive microenvironment in gliomas. Immunobiology 2020;225:151853.

93 Fecci PE, Mitchell DA, Whitesides JF, et al. Increased regulatory Tcell fraction amidst a diminished CD4 compartment explains cellular immune defects in patients with malignant glioma. Cancer Res 2006;66:3294-302.

94 Crane CA, Ahn BJ, Han SJ, et al. Soluble factors secreted by glioblastoma cell lines facilitate recruitment, survival, and expansion of regulatory T cells: implications for immunotherapy. Neuro Oncol 2012;14:584-95.

95 Jordan JT, Sun W, Hussain SF, et al. Preferential migration of regulatory $T$ cells mediated by glioma-secreted chemokines can be blocked with chemotherapy. Cancer Immunol Immunother 2008;57:123-31.

96 Darmanis S, Sloan SA, Croote D, et al. Single-Cell RNA-seq analysis of infiltrating neoplastic cells at the migrating front of human glioblastoma. Cell Rep 2017:21:1399-410.

97 Woroniecka K, Chongsathidkiet P, Rhodin K, et al. T-Cell exhaustion signatures vary with tumor type and are severe in glioblastoma. Clinical Cancer Research 2018;24:4175-86.

98 Kmiecik J, Poli A, Brons NHC, et al. Elevated $\mathrm{CD}^{+}$and $\mathrm{CD}^{+}$ tumor-infiltrating immune cells correlate with prolonged survival in glioblastoma patients despite integrated immunosuppressive mechanisms in the tumor microenvironment and at the systemic level. J Neuroimmunol 2013;264:71-83.

99 Park J, Kwon M, Kim KH, et al. Immune Checkpoint Inhibitorinduced Reinvigoration of Tumor-infiltrating CD8 ${ }^{+} \mathrm{T}$ Cells is Determined by Their Differentiation Status in Glioblastoma. Clin Cancer Res 2019;25:2549-59.

100 Rahman M, Kresak J, Yang C, et al. Analysis of immunobiologic markers in primary and recurrent glioblastoma. $J$ Neurooncol 2018;137:249-57

101 Mu L, Yang C, Gao Q, et al. Cd4 ${ }^{+}$and perivascular Foxp3 ${ }^{+} \mathrm{T}$ cells in glioma correlate with angiogenesis and tumor progression. Front Immunol 2017:8:1451.

102 Wood GW, Holladay FP, Turner T, et al. A pilot study of autologous cancer cell vaccination and cellular immunotherapy using antiCD3 stimulated lymphocytes in patients with recurrent grade III/IV astrocytoma. J Neurooncol 2000;48:113-20.

103 O'Rourke DM, Nasrallah MP, Desai A, et al. A single dose of peripherally infused EGFRvIll-directed CAR T cells mediates antigen loss and induces adaptive resistance in patients with recurrent glioblastoma. Sci Trans/ Med 2017;9:eaaa0984.

104 Wick W, Wick A, Sahm F, et al. VXM01 phase I study in patients with progressive glioblastoma: final results. JCO 2018;36:2017.

105 Dillman RO, Duma CM, Ellis RA, et al. Intralesional lymphokineactivated killer cells as adjuvant therapy for primary glioblastoma. $J$ Immunother 2009;32:914-9.

106 Lang FF, Conrad C, Gomez-Manzano C, et al. Phase I study of DNX-2401 (Delta-24-RGD) oncolytic adenovirus: replication and immunotherapeutic effects in recurrent malignant glioma. J Clin Oncol 2018;36:1419-27.

107 Wu A, Maxwell R, Xia Y, et al. Combination anti-CXCR4 and antiPD-1 immunotherapy provides survival benefit in glioblastoma through immune cell modulation of tumor microenvironment. $J$ Neurooncol 2019;143:241-9.

108 Inogés S, Tejada S, de Cerio AL-D, et al. A phase II trial of autologous dendritic cell vaccination and radiochemotherapy following fluorescence-guided surgery in newly diagnosed glioblastoma patients. J Trans/ Med 2017;15:104

109 Fenstermaker R. Vaccine therapy and Sargramostim in treating patients with malignant glioma, 2017. Available: https://clinicaltrials. $\mathrm{gov} / \mathrm{ct} 2 / \mathrm{show} / \mathrm{study} / \mathrm{NCT} 01250470$ ?term=immune+therapy\&recrs= e\&cond=Glioblastoma+Multiforme\&rank $=24$

110 Sampson JH, Heimberger AB, Archer GE, et al. Immunologic escape after prolonged progression-free survival with epidermal growth factor receptor variant III peptide vaccination in patients with newly diagnosed glioblastoma. J Clin Oncol 2010;28:4722-9.

111 Weller M, Butowski N, Tran DD, et al. Rindopepimut with temozolomide for patients with newly diagnosed, EGFRvIIIexpressing glioblastoma (act IV): a randomised, double-blind, International phase 3 trial. Lancet Oncol 2017;18:1373-85.

112 Sampson JH, Aldape KD, Archer GE, et al. Greater chemotherapyinduced lymphopenia enhances tumor-specific immune responses that eliminate EGFRvIll-expressing tumor cells in patients with glioblastoma. Neuro Oncol 2011;13:324-33.

113 Phuphanich S, Wheeler CJ, Rudnick JD, et al. Phase I trial of a multi-epitope-pulsed dendritic cell vaccine for patients with 
newly diagnosed glioblastoma. Cancer Immunol Immunother 2013;62:125-35.

114 Berghoff AS, Kiesel B, Widhalm G, et al. Programmed death ligand 1 expression and tumor-infiltrating lymphocytes in glioblastoma. Neuro Oncol 2015;17:1064-75.

115 Nduom EK, Wei J, Yaghi NK, et al. Pd-L1 expression and prognostic impact in glioblastoma. Neuro Oncol 2016;18:195-205.

116 Pratt D, Dominah G, Lobel G, et al. Programmed death ligand 1 is a negative prognostic marker in recurrent isocitrate DehydrogenaseWildtype glioblastoma. Neurosurgery 2019;85:280-9.

117 Omuro A, Vlahovic G, Baehring J, et al. OS07.3 nivolumab in combination with radiotherapy with or without temozolomide in patients with newly diagnosed glioblastoma: updated results from CheckMate 143. Neuro Oncol 2017;19:13.

118 Bloch O, Lim M, Sughrue ME, et al. Autologous heat shock protein peptide vaccination for newly diagnosed glioblastoma: impact of peripheral PD-L1 expression on response to therapy. Clin Cancer Res 2017;23:3575-84.

119 Stupp R, Mason WP, van den Bent MJ, et al. Radiotherapy plus concomitant and adjuvant temozolomide for glioblastoma. $N$ Engl $J$ Med 2005;352:987-96.

120 Gilbert MR, Dignam JJ, Armstrong TS, et al. A randomized trial of bevacizumab for newly diagnosed glioblastoma. N Engl J Med 2014;370:699-708.

121 Liau LM, Ashkan K, Tran DD, et al. First results on survival from a large phase 3 clinical trial of an autologous dendritic cell vaccine in newly diagnosed glioblastoma. J Transl Med 2018;16:142.

122 Zhao J, Chen AX, Gartrell RD, et al. Immune and genomic correlates of response to anti-PD-1 immunotherapy in glioblastoma. Nat Med 2019;25:462-9.

123 Bleeker FE, Atai NA, Lamba S, et al. The prognostic IDH1(R132) mutation is associated with reduced $\mathrm{NADP}^{+}$-dependent IDH activity in glioblastoma. Acta Neuropathol 2010;119:487-94.

124 Desjardins A, Gromeier M, Herndon JE, et al. Recurrent glioblastoma treated with recombinant poliovirus. $N$ Engl $J$ Med 2018;379:150-61.

125 Ülgen E, Can Özge, Bilguvar K, et al. Whole exome sequencingbased analysis to identify DNA damage repair deficiency as a major contributor to gliomagenesis in adult diffuse gliomas. J Neurosurg 2019:1-12.

126 Zhao Y, Carter R, Natarajan S, et al. Single-Cell RNA sequencing reveals the impact of chromosomal instability on glioblastoma cancer stem cells. BMC Med Genomics 2019;12:79.

127 Patel AP, Tirosh I, Trombetta JJ, et al. Single-Cell RNA-seq highlights intratumoral heterogeneity in primary glioblastoma. Science 2014;344:1396-401.

128 Akgül S, Patch A-M, D'Souza RCJ, et al. Intratumoural heterogeneity underlies distinct therapy responses and treatment resistance in glioblastoma. Cancers 2019;11. doi:10.3390/ cancers11020190. [Epub ahead of print: 06 Feb 2019].

129 Fasterius E, Uhlén M, Al-Khalili Szigyarto C. Single-Cell RNA-seq variant analysis for exploration of genetic heterogeneity in cancer. Sci Rep 2019;9:9524.

130 Peng S, Dhruv H, Armstrong B, et al. Integrated genomic analysis of survival outliers in glioblastoma. Neuro Oncol 2017;19:833-44.

131 Feng L, Qian H, Yu X, et al. Heterogeneity of tumor-infiltrating lymphocytes ascribed to local immune status rather than neoantigens by multi-omics analysis of glioblastoma multiforme. Sci Rep 2017;7:6968.

132 Bouffet E, Larouche V, Campbell BB, et al. Immune checkpoint inhibition for Hypermutant glioblastoma multiforme resulting from germline biallelic mismatch repair deficiency. J Clin Oncol 2016;34:2206-11.

133 Hodges TR, Ott M, Xiu J, et al. Mutational burden, immune checkpoint expression, and mismatch repair in glioma: implications for immune checkpoint immunotherapy. Neuro Oncol 2017;19:1047-57.

134 Belson A, Schmidt T, Fernando D, et al. Characterisation of the clinical and activated $\mathrm{T}$ cell response to repeat delayed-type hypersensitivity skin challenges in human subjects, with KLH and $\mathrm{PPD}$, as a potential model to test T cell-targeted therapies. Inflamm Res 2016;65:389-404.

135 Gedeon PC, Choi BD, Sampson JH, et al. Rindopepimut: antiEGFRvIll peptide vaccine, oncolytic. Drugs Future 2013;38:147-55.
136 Schneider T, Gerhards R, Kirches E, et al. Preliminary results of active specific immunization with modified tumor cell vaccine in glioblastoma multiforme. J Neurooncol 2001;53:39-46.

137 Dietrich P-Y. Phase I/II trial of IMA950 Multi-peptide vaccine plus Poly-ICLC in glioblastoma, 2016. Available: https://clinicaltrials.gov/ ct2/show/study/NCT01920191?term=immune+therapy\&recrs=e\& cond=Glioblastoma + Multiforme

138 Cloughesy TF, Mochizuki AY, Orpilla JR, et al. Neoadjuvant antiPD-1 immunotherapy promotes a survival benefit with intratumoral and systemic immune responses in recurrent glioblastoma. Nat Med 2019;25:477-86.

139 Schalper KA, Rodriguez-Ruiz ME, Diez-Valle R, et al. Neoadjuvant nivolumab modifies the tumor immune microenvironment in resectable glioblastoma. Nat Med 2019;25:470-6.

140 Weathers S-P, Gilbert MR. Current challenges in designing GBM trials for immunotherapy. J Neurooncol 2015;123:331-7.

141 Jackson S, Weingart J, Nduom EK, et al. The effect of an adenosine $A_{2 A}$ agonist on intra-tumoral concentrations of temozolomide in patients with recurrent glioblastoma. Fluids Barriers CNS 2018;15:2.

142 Sarrafzadeh A, Schlenk F, Gericke C, et al. Relevance of cerebral interleukin-6 after aneurysmal subarachnoid hemorrhage. Neurocrit Care 2010;13:339-46.

143 Hutchinson PJ, O'Connell MT, Rothwell NJ, et al. Inflammation in human brain injury: intracerebral concentrations of IL-1alpha, IL-1beta, and their endogenous inhibitor IL-1ra. J Neurotrauma 2007;24:1545-57

144 Haslene-Hox H, Madani A, Berg KCG, et al. Quantification of the concentration gradient of biomarkers between ovarian carcinoma interstitial fluid and blood. BBA Clin 2014;2:18-23.

145 Sedlaczek P, Frydecka I, Gabryś M, et al. Comparative analysis of CA125, tissue polypeptide specific antigen, and soluble interleukin-2 receptor alpha levels in sera, cyst, and ascitic fluids from patients with ovarian carcinoma. Cancer 2002;95:1886-93.

146 Simpson RJ, Bernhard OK, Greening DW, et al. Proteomics-driven cancer biomarker discovery: looking to the future. Curr Opin Chem Biol 2008;12:72-7.

147 Portnow J, Badie B, Liu X, et al. A pilot microdialysis study in brain tumor patients to assess changes in intracerebral cytokine levels after craniotomy and in response to treatment with a targeted anticancer agent. J Neurooncol 2014;118:169-77.

148 Tabatabaei P, Visse E, Bergström P, et al. Radiotherapy induces an immediate inflammatory reaction in malignant glioma: a clinical microdialysis study. J Neurooncol 2017;131:83-92.

149 Nduom EK. Cytokine microdialysis for real-time immune monitoring in glioblastoma patients undergoing checkpoint blockade, 2019. Available: https://clinicaltrials.gov/ct2/show/NCT03493932

150 Shigeyasu K, Toden S, Zumwalt TJ, et al. Emerging role of microRNAs as liquid biopsy biomarkers in gastrointestinal cancers. Clin Cancer Res 2017;23:2391-9.

151 Nordgård O, Tjensvoll K, Gilje B, et al. Circulating tumour cells and DNA as liquid biopsies in gastrointestinal cancer. $\mathrm{Br} J$ Surg 2018:105:e110-20.

152 Kitahara M, Hazama S, Tsunedomi R, et al. Prediction of the efficacy of immunotherapy by measuring the integrity of cell-free DNA in plasma in colorectal cancer. Cancer Sci 2016;107:1825-9.

153 Cabel L, Proudhon C, Romano E, et al. Clinical potential of circulating tumour DNA in patients receiving anticancer immunotherapy. Nat Rev Clin Oncol 2018;15:639-50.

154 Phallen J, Sausen M, Adleff V, et al. Direct detection of early-stage cancers using circulating tumor DNA. Sci Trans/ Med 2017;9. doi:10.1126/scitransImed.aan2415. [Epub ahead of print: 16 Aug 2017].

155 Matson V, Fessler J, Bao R, et al. The commensal microbiome is associated with anti-PD-1 efficacy in metastatic melanoma patients. Science 2018;359:104-8.

156 Antonios JP, Soto H, Everson RG, et al. Detection of immune responses after immunotherapy in glioblastoma using PET and MRI. Proc Natl Acad Sci U S A 2017;114:10220-5.

157 Rashidian M, LaFleur MW, Verschoor VL, et al. Immuno-PET identifies the myeloid compartment as a key contributor to the outcome of the antitumor response under PD-1 blockade. Proc Nat/ Acad Sci U S A 2019;116:16971-80. 